\title{
Positive Attitudes and Therapeutic Misconception Around Hypothetical Clinical Trial Participation in the Huntington's Disease Community
}

\author{
Kristina Cotter ${ }^{\mathrm{a}}$, Carly E. Siskind ${ }^{\mathrm{b}}$, Sharon J. Sha ${ }^{\mathrm{b}}$ and Andrea K. Hanson-Kahn ${ }^{\mathrm{a}, \mathrm{c}, *}$ \\ ${ }^{a}$ Department of Genetics, Stanford University School of Medicine, Stanford, CA, USA \\ ${ }^{\mathrm{b}}$ Department of Neurology and Neurological Sciences, Stanford University, Stanford, CA, USA \\ ${ }^{\mathrm{c}}$ Department of Pediatrics, Division of Medical Genetics, Stanford University Medical Center, Stanford, \\ CA, USA
}

\begin{abstract}
.
Background: New therapies that could modify the disease course of Huntington's disease (HD) are entering clinical trials. However, conceptions about clinical research from the HD community are unknown. This knowledge could help inform patient-clinician discussions surrounding clinical trial participation.

Objective: The purpose of this study was to assess clinical trial attitudes and understanding in the HD community.

Methods: We developed a survey incorporating two measures of trial understanding and attitudes and the impact of therapeutic route of administration on hypothetical trial participation. The survey was distributed via emails, flyers, and social media through HD-related organizations.

Results: There were 73 responses. Individuals self-reported as clinically diagnosed with HD, gene positive but asymptomatic, or primary caregivers. Respondents viewed clinical trials positively and generally viewed trials as safe. Individuals with prior HD-related research experience were less likely to have negative expectations about trials than those without research experience $(p=0.002)$, and women had higher information needs than men $(p=0.001)$. Individuals with HD were more likely than the other groups to experience therapeutic misconception $(p=0.002)$. All respondents were able to appraise risks and benefits of research but exhibited optimism about trial outcomes. Willingness to participate was highest when the route of administration was minimally invasive.

Conclusions: While the HD community views clinical trials positively, patients with HD are at high risk for therapeutic misconception and all groups are optimistic about trial outcomes. Limitations of this study include a small sample that may be inclined to view research positively given past trial participation and interest in participating in HD surveys. However, the findings from this study can be used to strengthen informed consent during HD clinical trial recruitment.
\end{abstract}

Keywords: Huntington's disease, clinical trial, clinical research, therapeutic misconception

\footnotetext{
${ }^{*}$ Correspondence to: Andrea Hanson-Kahn, Division of Medical Genetics, Department of Pediatrics, Stanford University Med-
} 


\section{INTRODUCTION}

Huntington's disease (HD) is an autosomal dominant, neurodegenerative disorder resulting in progressive motor skill deterioration, cognitive decline, and psychiatric disturbances [1]. HD is caused by a triplet repeat expansion in the huntingtin (HTT) gene that results in production of a mutant protein that accumulates in the striatum of the basal ganglia, leading to progressive neuronal dysfunction and death [2]. Current treatment for HD is focused on symptomatic management, though there are several potential disease-modifying therapeutic agents in development that could possibly modify the course of HD by decreasing the amount of mutant HTT protein in the brain. Reduction of mutant and total HTT protein in the central nervous system has been suggested to improve HD-related symptoms in several animal models. An antisense oligonucleotide (ASO) targeting HTT pre-mRNA is currently being tested in human clinical trials [3-6].

The prospect of disease-modifying therapies for HD offers hope after a long history of trial failures. Historically, only $3.5 \%$ of HD trials have progressed to the next phase [2]. However, it is not well understood what those in the HD community think or understand about clinical trials. Research examining views of the general public on various diseases suggests that positive views regarding clinical research generally correlate with motivation to participate in trials [7-10]. However, therapeutic misconception, wherein an individual does not understand that the primary purpose of research is to produce generalizable knowledge and not for their own personal benefit, is relatively common in the general public [9, 11-14]. Therapeutic misconception is concerning because it precludes the ability of a potential participant to provide truly informed consent [13, 15]. Misunderstanding of the research process can also result in therapeutic misestimation, in which an individual cannot realistically assess the benefits and risks of research participation, and can cause excessive optimism about outcomes (therapeutic optimism) [9, 13, 15].

There is concern that patients with severe or fatal conditions are susceptible to exposing themselves to higher research risks in hopes of finding a cure [16]. Members of the general population are more likely to indicate interest in trial participation if they are asked to imagine being diagnosed with a fatal disease [9]. Patients with severe neurodegenerative conditions such as amyotrophic lateral sclerosis (ALS) are less concerned with therapeutic side effects and trial burdens than those with less severe conditions and are highly optimistic about study outcomes [14, 17]. Studies in Parkinson's disease (PD) have suggested that patients with PD are at risk for therapeutic misconception [18]. Similar to ALS and PD, HD is a severe condition with minimal management options. A preliminary study of individuals in the HD community prior to the start of ASO trials found that while awareness of the clinical research process is low, willingness to participate is high [19]. A more recent study found that those in the HD community harbor positive views toward trials and find the prospect of improving their own quality of life to be a motivating factor for trial participation [20]. Given the unknown safety profile and invasive nature of current disease-modifying therapies in development for HD, it is critical to assess how individuals impacted by this condition weigh the benefits and risks of participating in research [21, 22].

In the coming years, the HD community will need to have an accurate understanding of the research process to make informed decisions regarding trial participation. Clinicians are responsible for discussing the outlook of HD research with patients, connecting families to trials, and managing expectations regarding trial outcomes [23, 24]. The present study sought to assess clinical trial attitudes and understanding in the HD community in order to help prepare clinicians for discussions about participation in HD clinical trials.

\section{MATERIALS AND METHODS}

This study was reviewed and approved by the Institutional Review Board at Stanford University.

\section{Participants}

The target population consisted of individuals who self-reported as clinically diagnosed with symptomatic HD (“diagnosed with HD”), gene positive but clinically asymptomatic ("gene positive"), or primary caregivers of an individual with HD. All respondents were over the age of 18 years, spoke English, and lived within the United States. All respondents reported completing the online survey without assistance from another individual.

\section{Recruitment}

A multifaceted recruitment approach was employed. The survey was advertised directly 
to potential participants via advertisements on HD-related websites (the Huntington's Disease Society of America (HDSA) research blog, HDSA survey website), social media (HDSA, HD Facebook groups), email listservs (HDSA and the Huntington's Disease Youth Organization), and flyers handed out at the HDSA Team Hope walk in San Francisco (October 2018) and the HD Clinic at Stanford Medical Center in Stanford, CA. The survey was also advertised via email to HDSA support group leaders and to clinicians who see families with HD via the Huntington Study Group listerv and the National Society of Genetic Counselors' Neurogenetics Special Interest Group online forum. Respondents were recruited between October 11, 2018 and January 26, 2019. Given the multifaceted nature of the recruitment effort, it is not possible to estimate how many people viewed the recruitment materials. While the study was advertised across the United States, the in-person recruitment was in Northern California, USA.

\section{Instrumentation}

A survey was developed using Qualtrics software to examine attitudes and understanding towards a hypothetical HD clinical trial. The survey was designed with input from two genetic counselors and one physician with clinical expertise in HD, piloted on a former caregiver of a patient with HD, and reviewed and edited by the HDSA. Items in the survey included relevant demographic variables (see Table 1). Primary caregivers were also asked to select how they are related to the individual with HD and whether they are at-risk for developing HD. To assess clinical trial attitudes and understanding, two measures were employed (the PACT 22 Clinical Trial Attitudes Scale and the Therapeutic Misunderstanding Scale). An adapted version of each scale, wherein each statement was made to be specific to HD, was employed for this study. Respondents were asked to imagine a hypothetical scenario in which they or the individual with HD were considering participation in a HD clinical trial. Respondents then were asked if they agreed with each statement on a five-point Likert scale. The statements were randomized for all respondents. Finally, respondents were asked to rate their willingness to participate in a clinical trial on a 5point Likert scale based on the route of administration of the hypothetical therapy (oral, nasal, injection or infusion into the arm, injection into the brain, injection into the spinal column, and injection into the eyeball).

\section{PACT 22 clinical trial attitudes scale}

The PACT 22 Clinical Trial Attitudes scale assesses patient views and attitudes towards clinical trials [25]. PACT 22 consists of 22 statements divided into five subscales with good internal consistency:

Table 1

Characteristics of survey respondents

\begin{tabular}{|c|c|c|c|c|c|c|c|c|}
\hline & \multicolumn{2}{|c|}{$\begin{array}{c}\text { Diagnosed } \\
\text { (symptomatic) }\end{array}$} & \multicolumn{2}{|c|}{$\begin{array}{l}\text { Gene positive } \\
\text { (asymptomatic) }\end{array}$} & \multicolumn{2}{|c|}{$\begin{array}{c}\text { Primary } \\
\text { caregivers }\end{array}$} & \multicolumn{2}{|c|}{ Total } \\
\hline & 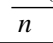 & $\%$ & $\bar{n}$ & $\%$ & $\bar{n}$ & $\%$ & 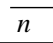 & $\%$ \\
\hline$n$ & 20 & $27.4 \%$ & 22 & $30.1 \%$ & 31 & $42.5 \%$ & 73 & $100.0 \%$ \\
\hline Female & 13 & $65.0 \%$ & 19 & $86.4 \%$ & 28 & $90.3 \%$ & 60 & $82.2 \%$ \\
\hline Age 50 or Older & 15 & $75.0 \%$ & 7 & $31.8 \%$ & 21 & $67.7 \%$ & 43 & $58.9 \%$ \\
\hline \multicolumn{9}{|l|}{ Geography } \\
\hline New England (CT, ME, MA, NH, RI, VT) & 5 & $25.0 \%$ & 1 & $4.5 \%$ & 3 & $9.7 \%$ & 9 & $12.3 \%$ \\
\hline Mid-Atlantic (NJ, NY, PA) & 4 & $20.0 \%$ & 3 & $13.6 \%$ & 5 & $16.1 \%$ & 12 & $16.4 \%$ \\
\hline Mid-Atlantic (DE, DC, FL, GA, MD, NC, SC, VA, WV) & 1 & $5.0 \%$ & 7 & $31.8 \%$ & 5 & $16.1 \%$ & 13 & $17.8 \%$ \\
\hline Midwest 2 (IN, IL, MI, OH, WI) & 4 & $20.0 \%$ & 3 & $13.6 \%$ & 3 & $9.7 \%$ & 10 & $13.7 \%$ \\
\hline Midwest 2 (IA, KS, MN, MO, NE, ND, SD) & 0 & $0.0 \%$ & 2 & $9.1 \%$ & 1 & $3.2 \%$ & 3 & $4.1 \%$ \\
\hline Southeast (AL, KY, MS, TN) & 1 & $5.0 \%$ & 0 & $0.0 \%$ & 5 & $16.1 \%$ & 6 & $8.2 \%$ \\
\hline South (AR, LA, OK, TX) & 0 & $0.0 \%$ & 2 & $9.1 \%$ & 2 & $6.5 \%$ & 4 & $5.5 \%$ \\
\hline Mountain (AZ, CO, ID, NM, MT, UT, NV, WY) & 0 & $0.0 \%$ & 0 & $0.0 \%$ & 1 & $3.2 \%$ & 1 & $1.4 \%$ \\
\hline Pacific (AK, CA, HI, OR, WA) & 5 & $25.0 \%$ & 4 & $18.2 \%$ & 6 & $19.4 \%$ & 15 & $20.5 \%$ \\
\hline Education: Bachelor's Degree or Above & 7 & $35.0 \%$ & 15 & $68.2 \%$ & 19 & $61.3 \%$ & 41 & $56.2 \%$ \\
\hline Past Participation in HD Research & 10 & $50.0 \%$ & 17 & $77.3 \%$ & 21 & $67.7 \%$ & 48 & $65.8 \%$ \\
\hline Past Participation in Non-HD Research & 4 & $20.0 \%$ & 0 & $0.0 \%$ & 3 & $9.7 \%$ & 7 & $9.6 \%$ \\
\hline \multicolumn{9}{|l|}{ Past Support Group Attendance } \\
\hline Yes, within the past year & 9 & $45.0 \%$ & 5 & $22.7 \%$ & 16 & $51.6 \%$ & 30 & $41.1 \%$ \\
\hline Yes, previous to the past year & 4 & $20.0 \%$ & 7 & $31.8 \%$ & 5 & $16.1 \%$ & 16 & $21.9 \%$ \\
\hline Never & 7 & $35.0 \%$ & 10 & $45.5 \%$ & 10 & $32.3 \%$ & 27 & $37.0 \%$ \\
\hline
\end{tabular}


Positive Beliefs, Safety, Information Needs, Negative Expectations, and Patient Involvement (Cronbach's alpha scores of 0.64-0.73). This scale was developed with patients diagnosed with cancer or asthma, and adaptations of this scale have been used to assess trial attitudes in PD and epilepsy [18, 26-28]. The scores from the scale were interpreted as follows: high Positive Beliefs scores suggested that respondents held positive views about trials; high Safety scores suggested respondents assumed clinical trial safeguards are in place, high Information Needs scores suggested respondents required information regarding trials, high Negative Expectations scores suggested respondents had negative expectations of taking part in trials, and high Patient Involvement scores suggested respondents believed patients should get involved in trials [25]. Permission to use the PACT 22 scale was granted by the copyright holder, the Picker Institute Europe, Oxford, UK.

\section{Therapeutic Misunderstanding Scale}

The Therapeutic Misunderstanding Scale (TMU) consists of 20 statements that measure three related factors: therapeutic misconception (failure to understand research methodology and that the primary purpose of research is to produce generalizable knowledge), therapeutic misestimation (overestimation of trial benefits or underestimation of trial risks), and therapeutic optimism (wherein respondents may understanding the research process, but remain highly hopeful about trial outcomes). The scale has good internal consistency (Cronbach's alpha scores of 0.75-0.83) [15]. Adaptations of this scale have been used to assess therapeutic misunderstanding in PD and epilepsy [18, 26, 27]. High scores (e.g., agreement) on each subscale indicated that an individual may be at-risk for therapeutic misconception, misestimation, or optimism, respectively [15]. Permission to use the TMU scale was granted by the author, Dr. Norm O'Rourke.

\section{Data analysis}

Data were analyzed using IBM SPSS version 25.0. For statistical analysis, each question was assessed based on the number of participants who completed the question. Responses were included for participants who completed both scales. Descriptive statistics were used to summarize all variables measured and are presented as mean \pm standard error of the mean (SEM) unless otherwise indicated. For sta- tistical analysis of the PACT 22 and TMU scales, each statement was assessed based on a numerical score between 1 and 5, with 1 representing "Strongly Disagree" and 5 representing "Strongly Agree." Cronbach's alpha was used to assess reliability within each subscale. Subscale scores were calculated via the means of each statement within the specific subscale $[15,25]$. Comparisons between groups were made using a Student's $t$ test for comparisons between two groups or one-way ANOVA with a Tukey HSD or Games-Howell post-test for comparison between three groups. As this study was initially designed as a descriptive study, a conservative value of $p<0.01$ was considered significant.

\section{RESULTS}

\section{Participant characteristics}

A total of 73 participants completed the survey. The demographics of the respondents are summarized in Table 1. Primary caregivers comprised the largest group $(42.5 \%)$, followed by gene positive asymptomatic individuals $(30.1 \%)$ and individuals with HD $(27.4 \%)$. The majority of respondents were female $(82.2 \%)$ and had participated in a HD-related clinical research in the past $(65.8 \%)$.

The majority of primary caregivers indicated that they care for their spouse or significant other $(61.3 \%)$, followed by their parent $(19.4 \%)$ or their child $(12.9 \%)$. A minority indicated that they care for their sibling or other family member $(6.4 \%)$. Of the 31 primary caregivers who completed the survey, $n=4(12.9 \%)$ were also at-risk to develop HD. $\mathrm{N}=4$ (18.2\%) of the 22 gene positive individuals were also caregivers.

\section{Clinical trial attitudes}

Clinical trial attitudes were evaluated using the PACT 22 scale (Supplementary Tables 1A-E) [25]. In general, respondents viewed clinical trial participation positively (Supplementary Tables 1A-E, Fig. 1A). The respondents held positive views about clinical trials $(M=4.33, S D=0.55)$ and did not have negative expectations of taking part in research $(M=2.46, S D=0.68)$. Respondents also believed that people with HD should participate in clinical trials $(M=4.17, S D=0.53)$ and expressed a preference for receiving information about the trial process before participation $(M=4.10, S D=0.58)$. Most respondents generally believed that safeguards are in place 



\section{Negative Expectations}

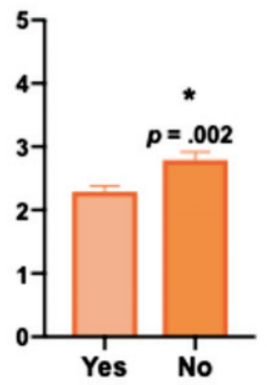

Past HD Research Experience?

Fig. 1. Attitudes toward hypothetical clinical trials in the HD community. A) The PACT 22 Clinical Trial Attitudes scale included 22 statements organized into five subscales: Positive Beliefs, Safety, Information Needs, Negative Expectations, and Patient Involvement [25]. Respondents were asked to rate their agreement with each statement on a five-point Likert scale, ranging from $1=$ Strongly Disagree to $5=$ Strongly Agree. Subscale scores were calculated via the means of each statement within the specific subscale. Comparisons between groups were made using a Student's $t$ test or one-way ANOVA. B) Females had higher information needs surrounding clinical trial participation $(M=4.20, S D=0.53)$ than males $(M=3.63, S D=0.55) ; t(71)=3.40, p=0.001$. C) Individuals with past HD research participation were less likely to have negative expectations about trial participation $(M=2.29, S D=0.62)$ than those without past research experience $(M=2.79$, $S D=0.67) ; t(71)=3.16, p=0.002$. The graphs shows average subscale scores \pm standard error of the mean (SEM).

to protect participants $(M=3.92, S D=0.52)$. There was good internal consistency for the Positive Beliefs $(\alpha=0.637)$, Information Needs $(\alpha=0.604)$, and Negative Expectations $(\alpha=0.732)$ subscales, but not for the Safety $(\alpha=0.368)$ and Patient Involvement $(\alpha=0.377)$ subscales.

The mean scores from each subscale were analyzed by the participant characteristics detailed in Table 1. One-way ANOVA did not identify any significant differences between the respondents' self-reported relationship to the HD community (diagnosed, gene positive, or primary caregiver) and attitudes toward clinical trials. Females had higher information needs surrounding clinical trial participation $(M=4.20, S D=0.53)$ than males $(M=3.63$, $S D=0.55) ; t(71)=3.40, p=0.001$ (Fig. 1B). Past participation in an HD observational study or clinical trial was also associated with trial attitudes. Indi- viduals with past HD research participation were significantly less likely to have negative expectations about trial participation $(M=2.29, S D=0.62)$ than those without past research experience $(M=2.79$, $S D=0.67) ; t(71)=3.16, p=0.002$ (Fig. 1C).

\section{Therapeutic misunderstanding}

Understanding of clinical research was evaluated using the Therapeutic Misunderstanding (TMU) scale (Supplemental Tables 2A-C). There was good internal consistency of responses for the Therapeutic Misconception $(\alpha=0.863)$, Therapeutic Misestimation $(\alpha=0.870)$, and Therapeutic Optimism $(\alpha=0.788)$ subscales. One-way ANOVA was conducted to compare responses between individuals who were diagnosed with HD, gene positive, or caregivers. There was a significant difference in 
the Therapeutic Misconception score between the three groups $[\mathrm{F}(2,70)=6.584, p=0.002]$ (Fig. 2). The Games Howell test indicated the mean score for individuals with HD $(M=3.44, S D=0.77)$ was significantly greater than both gene positive respondents $(M=2.62, S D=0.78, p<0.01)$ and caregivers $(M=2.71, S D=0.87, p<0.01)$. The scores between gene positive respondents and caregivers were not significantly different. Respondents with HD agreed with statements that suggested the main reason for research participation was for personal benefit and that the care during the course of a clinical trial was similar to that received during typical clinical care. In contrast, gene positive respondents and caregivers generally disagreed with these statements. In general, this indicates that individuals with HD are significantly more likely to experience therapeutic misconception than the other groups. Of note, all three groups agreed with the statement "this clinical trial is conducted mostly to gather knowledge about HD" (Supplementary Table 2A).

There were no significant differences in the Therapeutic Misestimation and Therapeutic Optimism scores between the three groups. Respondents from all three groups were able to correctly assess the risks and benefits of trial participation (therapeutic misestimation). Respondents also disagreed with the concept that trial participation would cure an individual's HD and expressed uncertainty about the guarantee of personal benefits to trial participation. Finally, all respondents were optimistic and hopeful about trial outcomes (Supplementary Tables B-C, Fig. 2). Mean TMU subscale scores were compared between the other participant characteristics collected in this study and no additional significant differences were identified.

\section{Impact of route of administration on trial participation}

The impact of therapeutic ROA on clinical trial participation was evaluated by asking respondents to rate how different ROAs would impact their willingness to participate in a hypothetical trial for HD. The most recent therapeutic agents in clinical development for HD are delivered via the spinal column [3]. The majority of participants indicated that they would participate if a therapy was administered orally, through the nostrils, or via injection or infusion in the arm. Willingness to participate decreased across the cohort when the ROA was an injection or infusion through the spinal column, brain, or eyeball
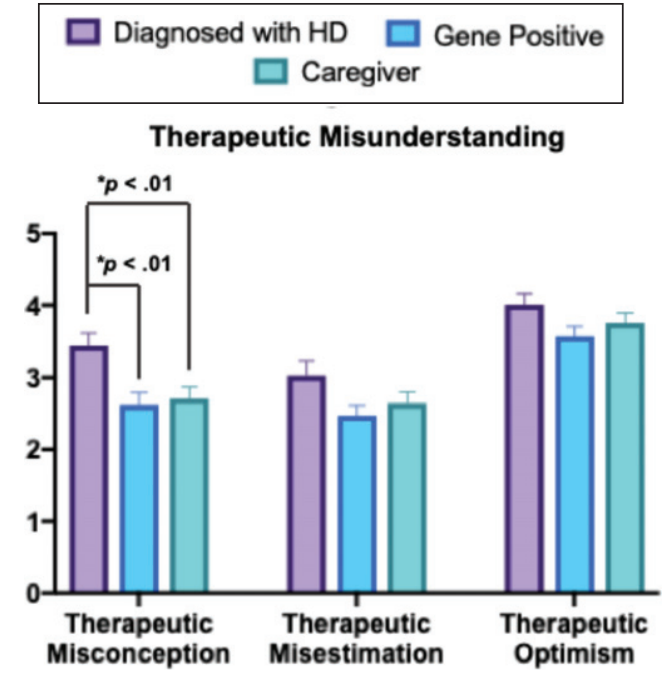

Fig. 2. Clinical trial understanding in the HD community. The TMU scale included 20 statements organize into three subscales: Therapeutic Misconception, Therapeutic Misestimation, and Therapeutic Optimism [15]. Respondents were asked to rate their agreement with each statement on a five-point Likert scale, ranging from $1=$ Strongly Disagree to $5=$ Strongly Agree. Subscale scores were calculated via the means of each statement within the specific subscale. Comparisons between groups were calculated using one-way ANOVA $[\mathrm{F}(2,70)=6.584, p=0.002]$ with the Games-Howell post-test. The mean score for individuals with HD $(M=3.44, S D=0.77)$ was significantly greater than both gene positive respondents $(M=2.62, S D=0.78, p<0.01)$ and caregivers $(M=2.71, S D=0.87, p<0.01)$ The graph shows average subscale scores \pm standard error of the mean (SEM). Asterisks $(*)$ indicate a significant difference compared to the other two groups.

(Fig. 3). Paired-samples $t$ tests indicated that willingness to participate was significantly lower when the ROA was invasive (spinal column, brain, or eyeball) relative to the noninvasive modalities (oral, nasal, or arm, $p<0.001)$. Among the invasive ROAs, respondents were least likely to participate if the ROA was via the brain or eyeball as compared to injection or infusion into the spinal column $(p<0.001)$. There were no significant differences in responses between the participant characteristic groups.

\section{DISCUSSION}

The results from this study reveal that individuals with HD, gene positive individuals, and caregivers harbor positive views about clinical research and believe that those with HD should participate. However, we also found that among the HD community, individuals with HD are at high risk for therapeutic misconception and the community as a whole 


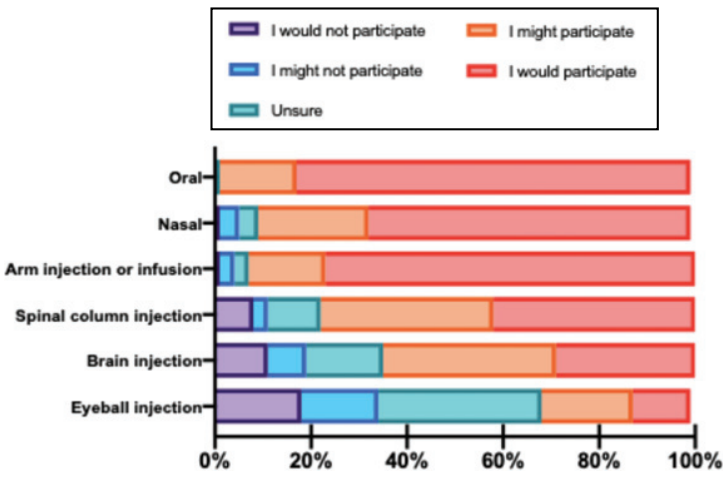

Fig. 3. Willingness to participate in clinical trial depends upon the invasiveness of the trial procedure. Respondents were asked to rate their willingness to participate in a hypothetical clinical trial that on a 5 -point Likert scale ( $1=\mathrm{I}$ would not participate to $5=\mathrm{I}$ would participate) based on the ROA of the therapy (oral, nasal, injection or infusion into the arm, or injection in the spinal column, brain, or eyeball). The graph represents the proportion of respondents who would or would not consider study participation for each possible ROA.

is highly optimistic about clinical trial outcomes. While this study is one of the first to assess trial attitudes and understanding in HD, our findings reflect research derived from patient populations with other neurological conditions, wherein trials are generally viewed positively and misconceptions surrounding research persist [7-10, 17, 18, 27]. Collectively, the results from this study have implications for both HD trial recruitment and the informed consent process.

We found that members of the HD community view clinical trial participation positively, similar to previous reports in both HD and PD [18, 20]. During initial development of the PACT 22 scale, patients with cancer and asthma were more likely to agree to participate in research if they had high Positive Beliefs, Patient Involvement, and Safety scores and a low Negative Expectations score [28]. Our research supports this pattern, suggesting those in the HD community are willing to take part in hypothetical research. However, our research also emphasized that our cohort desires as much information as possible about the research process before agreeing to participate, and this should be taken into account during trial recruitment researcher-participant conversations. Interestingly, females in our study had higher information needs than men. While this may indicate that females require more details about a clinical trial prior to providing consent, future work is required to explore this possibility given the small sample size of this study. There is limited data surrounding gender differences during trial recruitment and consent, and more research in this area is required. Of note, individuals who have previously been involved in HD-related research viewed the trial process more positively than individuals who were new to clinical research. Given this finding, clinicians and researchers may consider a patient's past experiences when discussing trial opportunities, noting that those with minimal research experience may have more concerns to address.

While attitudes regarding clinical trials were generally positive in our cohort, willingness to participate in research decreased as the invasive nature of a hypothetical intervention increased. These findings support recent research that invasive procedures decrease the likelihood an individual at risk for or diagnosed with HD will enroll in a clinical trial [20]. This is of interest given that upcoming clinical trials for HD involve therapeutics that are administered via injection into the spinal column (intrathecally) or into the brain $[3,6]$. Researchers should anticipate concerns about invasive procedures during trial recruitment and be prepared to discuss their associated burdens and risks with patients.

Patients affected by or at-risk for serious conditions may expose themselves to greater research risks in the hope of curing their illness or improving their quality of life $[13,16,17,21]$. Similar tendencies may underlie the positive attitudes of the HD population toward clinical research and trial participation [17]. It is therefore particularly important to address misunderstandings around the clinical research process with patients who have severe, fatal conditions in order to ensure that patients and their caregivers are making informed decisions about whether or not to take part in research. Our study found that individuals with HD were significantly more likely to experience therapeutic misconception than gene positive individuals or caregivers. Interestingly, those with HD generally agreed with the statement "this clinical trial is conducted mostly to gather knowledge about HD," but also agreed with statements suggesting they did not understand the difference between research and standard clinical care. This is concerning, because it draws into question whether the respondents with HD can consent to taking part in research versus pursuing standard of care only. However, it is reassuring that while all respondents in our study were highly optimistic about potential trial outcomes, they were generally able to make realistic assessments regarding possible risks and benefits of trial participation. 
It is not known why those with HD experienced a higher degree of therapeutic misconception than the other groups in this study. It appears contradictory that respondents with HD would understand the basic premise of research (to gather knowledge about HD) but score high on the misconception scale. Given that respondents with HD were able to assess risks and benefits of research, higher therapeutic misconception scores may represent a de-emphasis on the differences between standard of care and research that results from the fact that there are no diseasemodifying options available to patients with HD. This issue has been raised in clinical trials for children with severe, fatal diseases, wherein parents are at risk for therapeutic misconception because of the lack of alternative treatment options [29].

Though we were not able to evaluate the cognitive capabilities of the respondents, it is also possible that the impact of HD on cognition reduces the ability to make clear distinctions between standard of care and research. This raises the question of who should provide consent in HD research, particularly given the uncertainty of when cognitive symptoms of HD truly begin [1]. Past work in dementia and Alzheimer's disease supports a model in which those with mild to moderate cognitive impairment are involved in the decision-making process to enroll in a trial and provide assent, but that their surrogate decision maker be the one to provide consent [21,30-32]. There are few studies that evaluate the consent process in HD. One study of individuals with HD and their surrogate decision makers evaluating longitudinal comprehension of consent over the course of a trial found that both individuals with HD and their decision makers understood the nature of research and had similar comprehension scores at enrollment. However, these scores decreased in participants with HD over a year. This led the authors to recommend that individuals with HD provide consent if initial evaluations suggest comprehension of the research process, but that a surrogate decision maker step in when appropriate [21].

Overall, there is limited research available that evaluates therapeutic misconception in patients with or at-risk for cognitive impairment and in their surrogate decision makers. It is also challenging to assess when an individual is no longer able to provide their own consent $[32,33]$. The finding that those with HD are at a high risk for therapeutic misconception supports the need for more research in this area. Our results suggest that clinicians and researchers should take extra care to mitigate misconceptions about research with individuals who have HD. This includes spending time discussing the clinical trial process and purpose of research with HD patients. Given the high degree of optimism in our study population as a whole, we also recommend that these conversations set realistic expectations about the outcomes of research in order to mitigate future disappointment and assist in the decision-making process. We expect that those in the HD community will be open to in-depth conversations about trial participation in both the clinical and research settings, given the high information needs identified in this study. Finally, we agree with the recommendations set forth by De Langavant et al. that investigators should evaluate HD patients for comprehension of the study material, consent, and decision-making capability during enrollment and during the trial itself [21].

There are several limitations to this study. First, the sample was small and predominantly female, and the study was advertised via organizations that promote awareness of HD research. The study population was therefore likely more familiar with the research process than the broader HD community. Two-thirds of our study population had also previously taken part in an HD-related research study, which may increase the likelihood that the respondents were aware of the clinical trial process, limiting generalizability of the results. Furthermore, individuals who view trials negatively, who have had negative experiences in research, or who generally do not participate in clinical trials may have been less likely to respond to an advertisement for an HD-related survey. The respondents' interest in participating in this research was itself a source of bias, as the willingness to participate may indicate a higher likelihood to have positive views toward HD-related research. Second, respondents were also asked to self-report their diagnosed, gene positive, or caregiver status and we were not able to verify the cognitive status of the respondents. Additional research is required to evaluate the impact that cognitive status has on clinical trial attitudes and understanding. Many individuals in the HD community could also self-identify with more than one of the categories analyzed in this study; the sample size was not large enough to examine the impact that these factors had on study responses. Finally, respondents were asked to anticipate how they would feel in hypothetical situations surrounding clinical trial participation, and they may feel differently when faced with the actual choice to participate in research. Further research could explore whether receptiveness to hypothetical research participation holds true among 
a more representative sample of the community or those who are asked to enroll in an actual clinical trial.

In conclusion, this study suggests that the HD community views clinical trials positively and is interested in participating in therapeutic research. Attitudes toward clinical trials and willingness to participate may be influenced by past experience with HD research and the invasiveness of the study procedure. While the population as a whole is interested to take part in HD research, individuals with HD are at high risk for therapeutic misconception and the community appears highly optimistic about the outcomes of HD research. The findings from this study can be used to improve HD clinical trial recruitment and strengthen the informed consent process (Box 1). Clinicians and researchers should take extra care to ensure that those with HD understand the goals of research and should set realistic expectations about the outcomes of research with all involved.

Box 1. Recommendations for HD Investigators
1. Emphasize the distinction between research
and clinical standard of care; consider a patient's
past experiences with clinical research when dis-
cussing trial opportunities
2. Evaluate patients and caregivers for misunder-
standings around the clinical research process
3. Anticipate questions and concerns surround-
ing more invasive ROAs
4. Provide patients \& caregivers with both writ-
ten \& verbal information about the study; keep
in mind that females may require more details
than males

\section{ACKNOWLEDGMENTS}

This research was completed in partial fulfillment of a Masters in Genetic Counseling degree. This research was funded by a grant awarded to Kristina Cotter from the National Society of Genetic Counselors Neurogenetics Special Interest Group. The authors would also like to thank HDSA, HDYO, the Huntington Study Group, Stanford Medical Center, the National Society of Genetic Counselors, and the broader HD community for their help in recruiting respondents for this study.

\section{CONFLICTS OF INTEREST}

Carly Siskind has no conflicts of interest to report. Kristina Cotter is an employee of RDMD, Inc. Sharon
Sha receives research support from Biogen Idec, F Hoffmann-La Roche Ltd, Genentech, and Novartis for her role as Investigator in Clinical Trials. Dr. Sha has been a consultant for Abelson Taylor, Baird, Clearview Healthcare Partners, SelfCare Catalysts Inc, and the University of Southern California. Dr. Sha is funded by National Institutes of Health grants P50 AG047366 (Stanford Alzheimer's Disease Center) and R01 AG048076-02. Andrea Hanson-Kahn is a support group facilitator and board member for the Northern California Huntington Disease association.

\section{SUPPLEMENTARY MATERIAL}

The supplementary material is available in the electronic version of this article: http://dx.doi.org/ 10.3233/JHD-190382.

\section{REFERENCES}

[1] Ross CA, Aylward EH, Wild EJ, Langbehn DR, Long JD, Warner JH, et al. Huntington disease: Natural history, biomarkers and prospects for therapeutics. Nat Rev Neurol. 2014;10(4):204-16.

[2] McColgan P, Tabrizi SJ. Huntington's disease: A clinical review. Eur J Neurol. 2018;25(1):24-34.

[3] Rodrigues FB, Wild EJ. Clinical trials corner: September 2017. J Huntingtons Dis. 2017;6(3):255-63.

[4] Keiser MS, Kordasiewicz HB, McBride JL. Gene suppression strategies for dominantly inherited neurodegenerative diseases: Lessons from Huntington's disease and spinocerebellar ataxia. Hum Mol Genet. 2016;25(R1):R53-64.

[5] Abeliovich A, Manuscript A, Edward B. Lee, Virginia M.Y. Lee and JQT, Kordasiewicz HB, Stanek LM, Wancewicz EV, et al. Sustained therapeutic reversal of Huntington's disease by transient repression of huntingtin synthesis. Nat Rev Neurosci. 2013;13(1):38-50.

[6] Tabrizi S, Leavitt B, Landwehrmeyer B, Wild EJ, Saft $\mathrm{C}$, Barker $\mathrm{R}$, et al. Targeting huntingtin expression in patients with Huntington's disease. N Engl J Med. 2019;380(24):2307-16.

[7] Chu SH, Kim EJ, Jeong SH, Park GL. Factors associated with willingness to participate in clinical trials: A nationwide survey study. BMC Public Health. 2015;15(1):1-8.

[8] Madsen S, Holm S, Riis P. Ethical aspects of clinical trials: The attitudes of the public and out- patients. J Intern Med. 1999;245(6):571-9.

[9] Burns KEA, Magyarody N, Jiang D, Wald R. Attitudes and views of the general public towards research participation. Intern Med J. 2013;43(5):531-40.

[10] Jones CW, Braz VA, McBride SM, Roberts BW, Platts-Mills TF. Cross-sectional assessment of patient attitudes towards participation in clinical trials: Does making results publicly available matter? BMJ Open. 2016;6(11):1-6.

[11] Appelbaum P, Anatchkova M, Albert K, Dunn L, Lidz C. Therapeutic misconception in research subjects: Development and validation of a measure. Ann Intern Med. 2012;9(6):748-61.

[12] Ellis PM, Dowsett SM, Butow PN, Tattersall MHN. Attitudes to randomized clinical trials amongst out-patients 
attending a medical oncology clinic. Health Expect. 1999;2(1):33-43.

[13] Horng S, Grady C. Misunderstanding in clinical research: Distinguishing therapeutic misconception, therapeutic misestimation, \& therapeutic optimism. IRB Ethics Hum Res. 2003;25(1):11-6.

[14] Kim SYH, Wilson R, De Vries R, Ryan KA, Holloway RG, Kieburtz K. Are patients with amyotrophic lateral sclerosis at risk of a therapeutic misconception? J Med Ethics. 2016;42(8):514-8.

[15] Chou PHB, O'Rourke N. Development and initial validation of the Therapeutic Misunderstanding Scale for use with clinical trials research participants. Aging Ment Health. 2012;16(2):145-53.

[16] Kim SYH, Schrock L, Wilson R, Frank S, Holloway R, Kieburtz K, et al. An approach to evaluating therapeutic misconception. IRB Ethics Hum Res. 2009;31(5):7-14.

[17] DasMahapatra P, Raja P, Gilbert J, Wicks P. Clinical trials from the patient perspective: Survey in an online patient community. BMC Health Serv Res. 2017;17(1):166.

[18] Reijula E, Pietilä A, Halkoaho A, Selander T, Martikainen $\mathrm{K}$, Kälviäinen R, et al. Clinical features of Parkinson's disease patients are associated with therapeutic misconception and willingness to participate in clinical trials. Trials. 2017;18(444):1-9.

[19] Veatch Goodman L, Giuliano J, Lovecky D. Poster 1: Survey of clinical trial interest and literacy in Huntington support groups: Northwest pilot project. Neurotherapeutics. 2009;6(1):204.

[20] Bardakjian TM, Naczi KF, Gonzalez-Alegre P. Attitudes of potential participants towards molecular therapy trials in Huntington's disease. J Huntingtons Dis. 2019;8:1-7.

[21] De Langavant LC, Sudraud S, Verny C, Krystkowiak P, Simonin C, Damier P, et al. Longitudinal study of informed consent in innovative therapy research: Experience and provisional recommendations from a multicenter trial of intracerebral grafting. PLoS One. 2015;10(5):1-11.

[22] Sampaio C, Levey J, Klitzman R. Predictive testing and clinical trials in Huntington's disease: An ethical analysis: Ethics Of Testing in HD Trials. Mov Disord. 2018;33(2):243-7.

[23] Nance MA. Genetic counseling and testing for Huntington's disease: A historical review. Am J Med Genet B Neuropsychiatr Genet. 2017;174(1):75-92.
[24] Baig SS, Strong M, Rosser E, Taverner N V., Glew R, Miedzybrodzka Z, et al. 22 Years of predictive testing for Huntington's disease: The experience of the UK Huntington's Prediction Consortium. Eur J Hum Genet. 2016;24(10):1396-402.

[25] Jenkinson C, Burton JS, Cartwright J, Magee H, Hall I, Alcock C, et al. Patient attitudes to clinical trials: Development of a questionnaire and results from asthma and cancer patients. Health Expect. 2005;8(3):244-52.

[26] Reijula E, Halkoaho A, Pietilä A-M, Selander T, Martikainen K, Kälviäinen R, et al. Comparable indicators of therapeutic misconception between epilepsy or Parkinson's disease patients between those with clinical trial experience and trial non-participants. Seizure. 2018;60:61-7.

[27] Reijula E, Halkoaho A, Pietilä AM, Selander T, Kälviäinen $\mathrm{R}$, Keränen T. Therapeutic misconception correlates with willingness to participate in clinical drug trials among patients with epilepsy; need for better counseling. Epilepsy Behav. 2015;48(2015):29-34.

[28] Jenkinson C, Burton JS, Cartwright J, Magee H, Hall I, Alcock C, et al. Patient attitudes to clinical trials: Development of a questionnaire and results from asthma and cancer patients. Health Expect. 2005;8(3):244-52.

[29] Arkin L, Sondhi D, Worgall S, Suh LHK, Hackett N, Kaminsky S, et al. Confronting the issues of therapeutic misconception, enrollment decisions, and personal motives in genetic medicine-based clinical research studies for fatal disorders. Hum Gene Ther. 2005;16:1028-36.

[30] Black BS, Wechsler M, Fogarty L. Decision making for participation in dementia research. Am J Geriatr Psychiatry. 2013;21(4):355-63.

[31] Dunn LB, Fisher SR, Hantke M, Appelbaum PS, Dohan D, Young JP, et al. "Thinking about it for somebody else": Alzheimer's disease research and proxy decision makers' translation of ethical principles into practice. Am J Geriatr Psychiatry. 2013;21(4):337-45.

[32] Dunn LB, Palmer BW. When does therapeutic misconception affect surrogates' or subjects' decision making about whether to participate in dementia research? AMA J Ethics. 2017;19(7):678-85.

[33] Scott Kim, Caine ED, Currier GW, Leibovici A, Ryan JM. Assessing the competence of persons with Alzheimer's for participation in research. Am J Psychiatry. 2001;158(5):712-7. 